\title{
How to study runs of homozygosity using PLINK? A guide for analyzing medium density SNP data in livestock and pet species
}

\author{
R. Meyermans ${ }^{\dagger}$, W. Gorssen ${ }^{\dagger}$, N. Buys and S. Janssens ${ }^{*}$ (D)
}

\begin{abstract}
Background: PLINK is probably the most used program for analyzing SNP genotypes and runs of homozygosity $(\mathrm{ROH})$, both in human and in animal populations. The last decade, $\mathrm{ROH}$ analyses have become the state-of-the-art method for inbreeding assessment. In PLINK, the --homozyg function is used to perform ROH analyses and relies on several input settings. These settings can have a large impact on the outcome and default values are not always appropriate for medium density SNP array data. Guidelines for a robust and uniform ROH analysis in PLINK using medium density data are lacking, albeit these guidelines are vital for comparing different ROH studies. In this study, 8 populations of different livestock and pet species are used to demonstrate the importance of PLINK input settings. Moreover, the effects of pruning SNPs for low minor allele frequencies and linkage disequilibrium on $\mathrm{ROH}$ detection are shown.

Results: We introduce the genome coverage parameter to appropriately estimate $\mathrm{F}_{\mathrm{ROH}}$ and to check the validity of $\mathrm{ROH}$ analyses. The effect of pruning for linkage disequilibrium and low minor allele frequencies on $\mathrm{ROH}$ analyses is highly population dependent and such pruning may result in missed ROH. PLINK's minimal density requirement is crucial for medium density genotypes and if set too low, genome coverage of the $\mathrm{ROH}$ analysis is limited. Finally, we provide recommendations for the maximal gap, scanning window length and threshold settings.

Conclusions: In this study, we present guidelines for an adequate and robust ROH analysis in PLINK on medium density SNP data. Furthermore, we advise to report parameter settings in publications, and to validate them prior to analysis. Moreover, we encourage authors to report genome coverage to reflect the $\mathrm{ROH}$ analysis' validity. Implementing these guidelines will substantially improve the overall quality and uniformity of $\mathrm{ROH}$ analyses.
\end{abstract}

Keywords: PLINK, Runs of homozygosity, Minor allele frequency, Linkage disequilibrium, SNP density

\section{Background}

Runs of homozygosity ( $\mathrm{ROH})$ are the state-of-the-art method for inbreeding analyses in livestock populations [1]. ROHs are defined as long continuous homozygous stretches in the genome, which are - due to their length - assumed to arise from a common ancestor [2]. Whereas short $\mathrm{ROH}$ are indicators of distant inbreeding,

\footnotetext{
* Correspondence: steven.janssens@kuleuven.be

${ }^{\dagger}$ Roel Meyermans and Wim Gorssen shared first authorship.

Department of Biosystems, Livestock Genetics, KU Leuven, Kasteelpark

Arenberg 30 - Box 2472, 3001 Leuven, Belgium
}

long $\mathrm{ROH}$ suggest recent inbreeding [3]. $\mathrm{ROH}$ were first identified by Broman and Weber in the human genome, whereas Gibson et al. acknowledged their importance for inbreeding calculations $[4,5]$. McQuillan et al. defined the genomic inbreeding coefficient based on $\mathrm{ROH}$ $\left(\mathrm{F}_{\mathrm{ROH}}\right)[6]$.

PLINK $[7,8]$ is the most used program for $\mathrm{ROH}$ analyses in livestock populations [1]. $\mathrm{ROH}$ analyses are performed using the --homozyg function. The PLINK

(c) The Author(s). 2020 Open Access This article is distributed under the terms of the Creative Commons Attribution 4.0 International License (http://creativecommons.org/licenses/by/4.0/), which permits unrestricted use, distribution, and reproduction in any medium, provided you give appropriate credit to the original author(s) and the source, provide a link to the Creative Commons license, and indicate if changes were made. The Creative Commons Public Domain Dedication waiver (http://creativecommons.org/publicdomain/zero/1.0/) applies to the data made available in this article, unless otherwise stated. 
algorithm for $\mathrm{ROH}$ detection relies on a scanning window approach which roughly consists of three steps.

First, the scanning window is defined by a predefined number of SNPs (--homozyg-window-snp) with a maximal number of heterozygous SNPs (--homozyg-windowhet) and a maximal number of missing SNPs (--homozyg-window-missing). The defined window stepwise scans an individual's genome and scores for each SNP the proportion it appears in a homozygous window.

Second, segments of homozygous SNPs are identified genome wide by using a threshold for these scores per SNP: the scanning window hit rate (--homozyg-window-threshold). For a window size of 100 SNPs and a threshold of 0.05, a SNP has to appear in at least five homozygous windows before it is identified as part of a segment. Note that such homozygous windows may contain missing or heterozygous SNPs, depending on scanning window settings.

Third, extra constraints are set to these homozygous segments to identify the final $\mathrm{ROH}$ segments. The maximal interval between two SNPs in a segment is checked (--homozyg-gap) as well as the maximal amount of heterozygous SNPs allowed in the final $\mathrm{ROH}$ segment (--homozyg-het). Next, $\mathrm{ROH}$ segments that do not meet these two requirements are split and re-evaluated. This may lead to detecting $\mathrm{ROH}$ segments smaller than the scanning window size. Thereafter, the minimal SNP density (in $\mathrm{kb} / \mathrm{SNP}$ ) per segment is evaluated (--homo$z y g$-density) as well as the minimal length and number of SNPs (--homozyg-kb and --homozyg-snp). ROH segments which do not fulfill any of these three conditions are removed.

In literature, there is no consensus whether SNP data should be pruned for linkage disequilibrium (LD) and/or minor allele frequency (MAF) before $\mathrm{ROH}$ analysis. In Table 1 we provide an overview of recent $\mathrm{ROH}$ studies on medium density genotypes using PLINK. Most studies apply MAF pruning with a threshold between 0.010.05 and some studies also perform LD pruning. For example, Bjelland et al. and Zhang et al. prune all SNPs with $R^{2}>0.5$ (using bins of $50 \mathrm{SNPs}$ ), resulting in a reduced set of 7997 and 14,366 SNPs (unpruned > 50,000 SNPs), respectively $[11,15]$. Hence, this LD pruning results in a SNP reduction of more than $70 \%$.

The effect of minimal $\mathrm{ROH}$ length, either by the minimal number of SNPs or minimal kb length, has been thoroughly studied by Purfield et al. and Ferenčaković et al. [33, 34]. Purfield et al. concluded that a $50 \mathrm{~K} \mathrm{SNP}$ array is suitable for identifying ROHs longer than $5 \mathrm{Mb}$, whereas Ferenčaković et al. reasoned that the minimal $\mathrm{ROH}$ length should be adapted to the SNP density. They also found that heterozygous calls should be tolerated depending on the ROH length and SNP density [34]. Note that when allowing more than one heterozygous
SNP in a scanning window, adjacent heterozygous SNPs may cause the merging of different homozygous segments which are longer than the original ones.

Howrigan et al. simulated genotypes to test PLINK's $\mathrm{ROH}$ detection ability and varied several PLINK detection settings (--homozyg-window-snp, --homozygwindow-het, --homozyg-window-missing, --homozygwindow-threshold, --homozyg-snp) [35]. They concluded that data should be pruned for LD and MAF prior to analysis. However, Howrigan and colleagues did not vary scanning window sizes, maximal gap sizes, minimal density requirements (in $\mathrm{kb} / \mathrm{SNP}$ ) nor final $\mathrm{ROH}$ length in $\mathrm{kb}$, although these parameters can affect the outcome [35].

There is a large variation in parameter settings considering the maximal gap, minimal density and the scanning window size (Table 1). Moreover, studies often do not report density, gap and/or window size settings. Both Howrigan et al. and Peripolli et al. underlined a lack of consensus criteria for $\mathrm{ROH}$ analyses [1,35]. This lack of consensus will lead to biased results and hinders the comparison of results across studies.

In this paper, we provide guidelines for choosing PLINK parameter settings that ensure a robust and reliable $\mathrm{ROH}$ analysis. We used medium density genotypes in eight different livestock and pet species (pig, cattle, sheep, cats, horses, goats, dogs and chicken). First, we evaluated the effect of MAF and LD pruning on $\mathrm{ROH}$ analysis. Second, we investigated effects of the minimal density (--homozyg-density), the maximal interval between two SNPs in a ROH (-homozyg-gap), scanning window size (--homozyg-window-snp) and scanning window hit rate (--homozyg-window-threshold). Third, we introduce the genome coverage parameter to evaluate the validity of the $\mathrm{ROH}$ analysis and to estimate inbreeding based on $\mathrm{ROH}$ more accurately. These guidelines facilitate an adequate and robust $\mathrm{ROH}$ analysis, resulting in a higher overall quality and uniformity across studies.

\section{Results}

All analyses were performed on the eight different livestock and pet breeds. Results and figures for PIT, BB, MER and BUR are provided in the main manuscript, whereas results for SAA, ICE, LAB and BAR can be found in Additional files 1, 2, 3, 4, 5, 6 and 7 .

\section{Pruning for linkage disequilibrium}

The results of pruning for varying LD levels prior to $\mathrm{ROH}$ analysis for PIT, BB, MER and BUR are shown in Fig. 1, results for SAA, ICE, LAB and BAR are added in Additional file 2: Figure S1. The effects of LD pruning on the outcome of the $\mathrm{ROH}$ analysis was population dependent. Although maximal genome coverage was reached at $R^{2}>0.25$ in some populations (e.g. $\mathrm{BB}$ ), not 


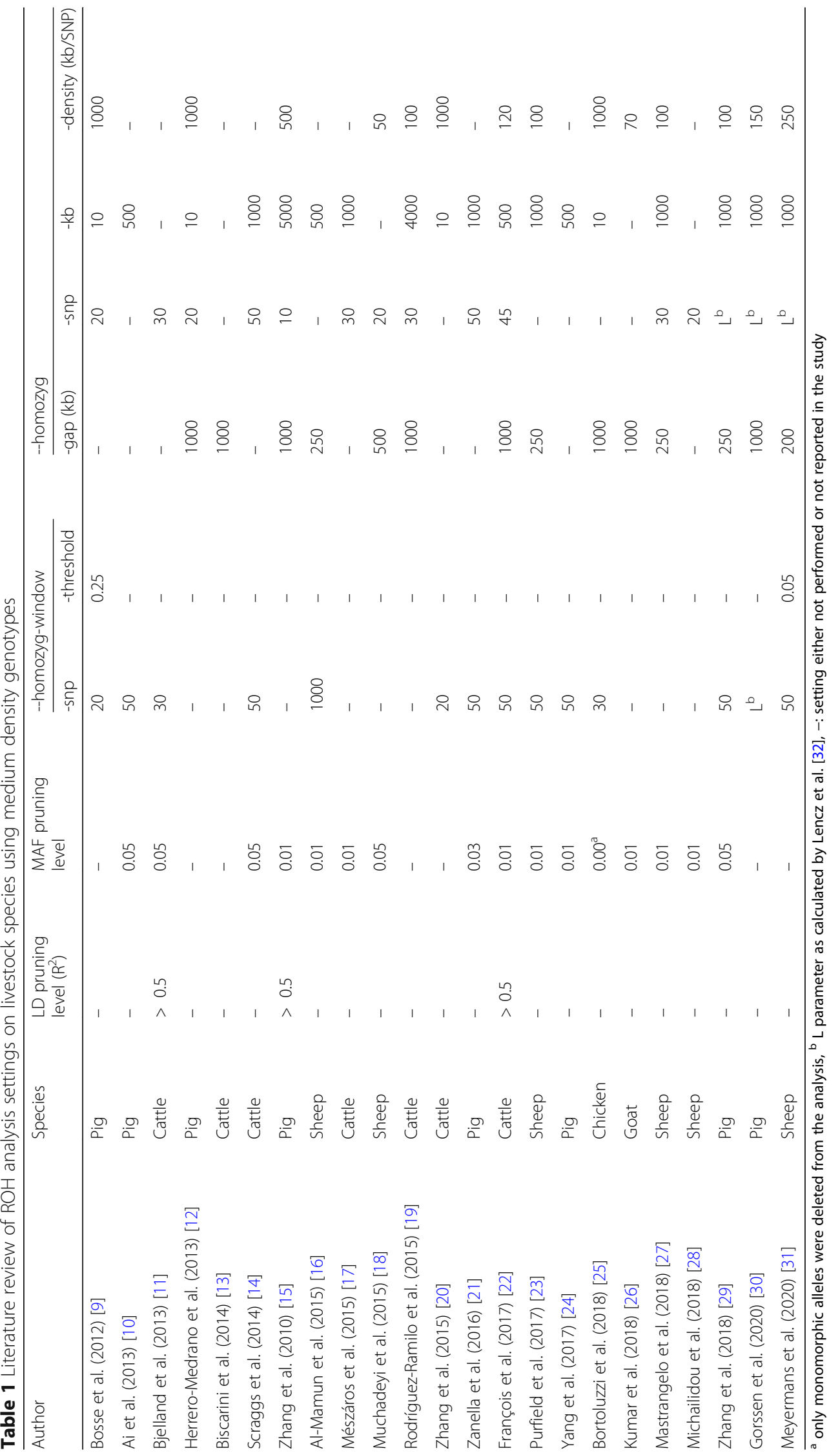




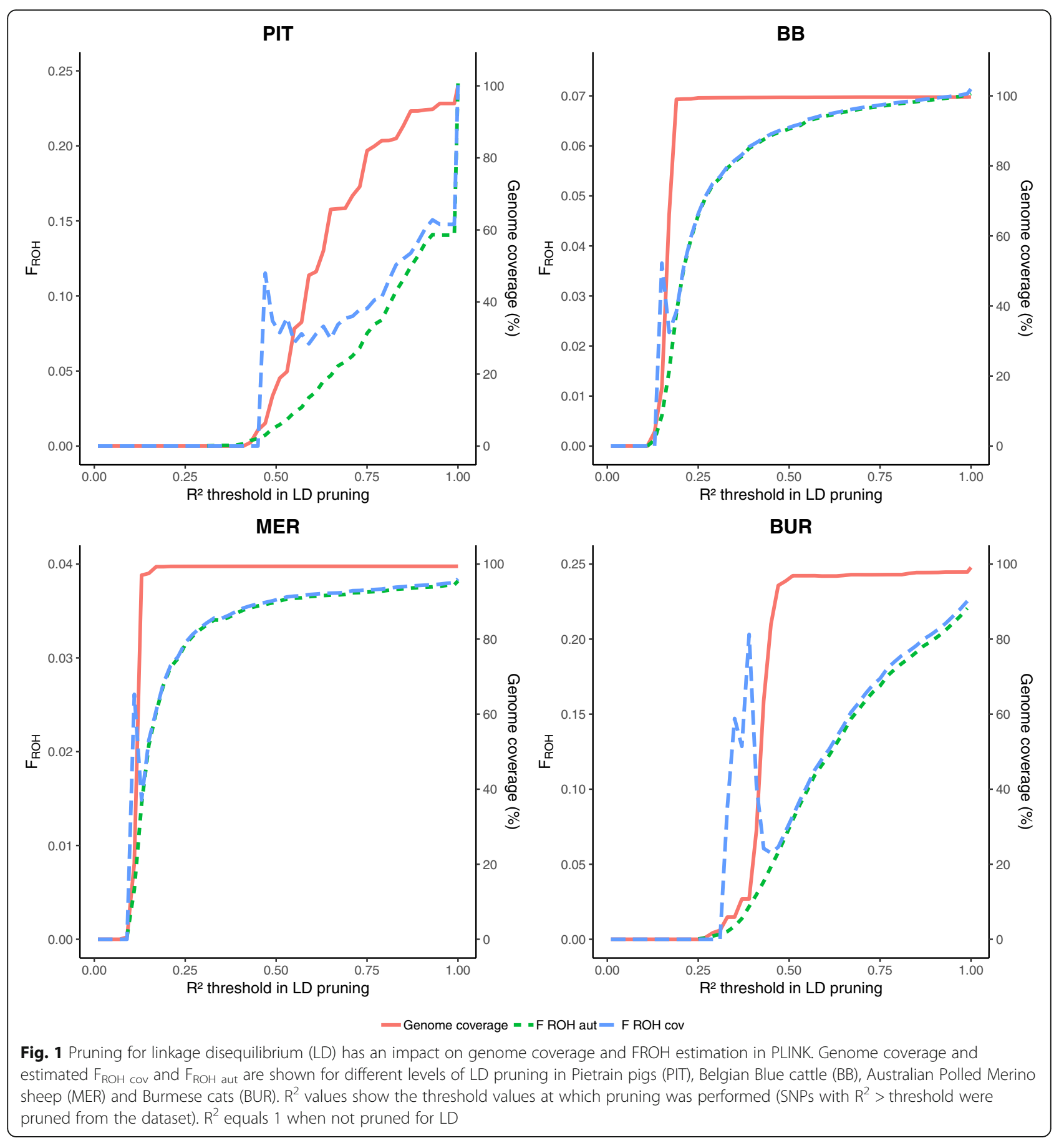

all $\mathrm{ROH}$ were detected and $\mathrm{F}_{\mathrm{ROH}}$ estimates were lower than without pruning for LD. In PIT, maximal genome coverage was reached more slowly in comparison to other populations (e.g. BB).

\section{Pruning for minor allele frequency}

In PIT and BUR, we observed that even mild MAF pruning (0.01) had an impact on $\mathrm{ROH}$ detection in several genomic regions. Figure 2 shows $\mathrm{ROH}$ incidence per
SNP (in \% of the total population) for both populations without MAF pruning (left) and with MAF pruning at 0.01 (right). For PIT, ROH islands were observed on SSC8 and SSC18, whereas for BUR, a change in observed $\mathrm{ROH}$ was found on e.g. B3, D1 and D3. These $\mathrm{ROH}$ in PIT and BUR would not have been detected if MAF pruning was performed. For the six other populations, little differences were observed in genome coverage and $\mathrm{F}_{\mathrm{ROH}}$ estimates by varying MAF pruning levels. 

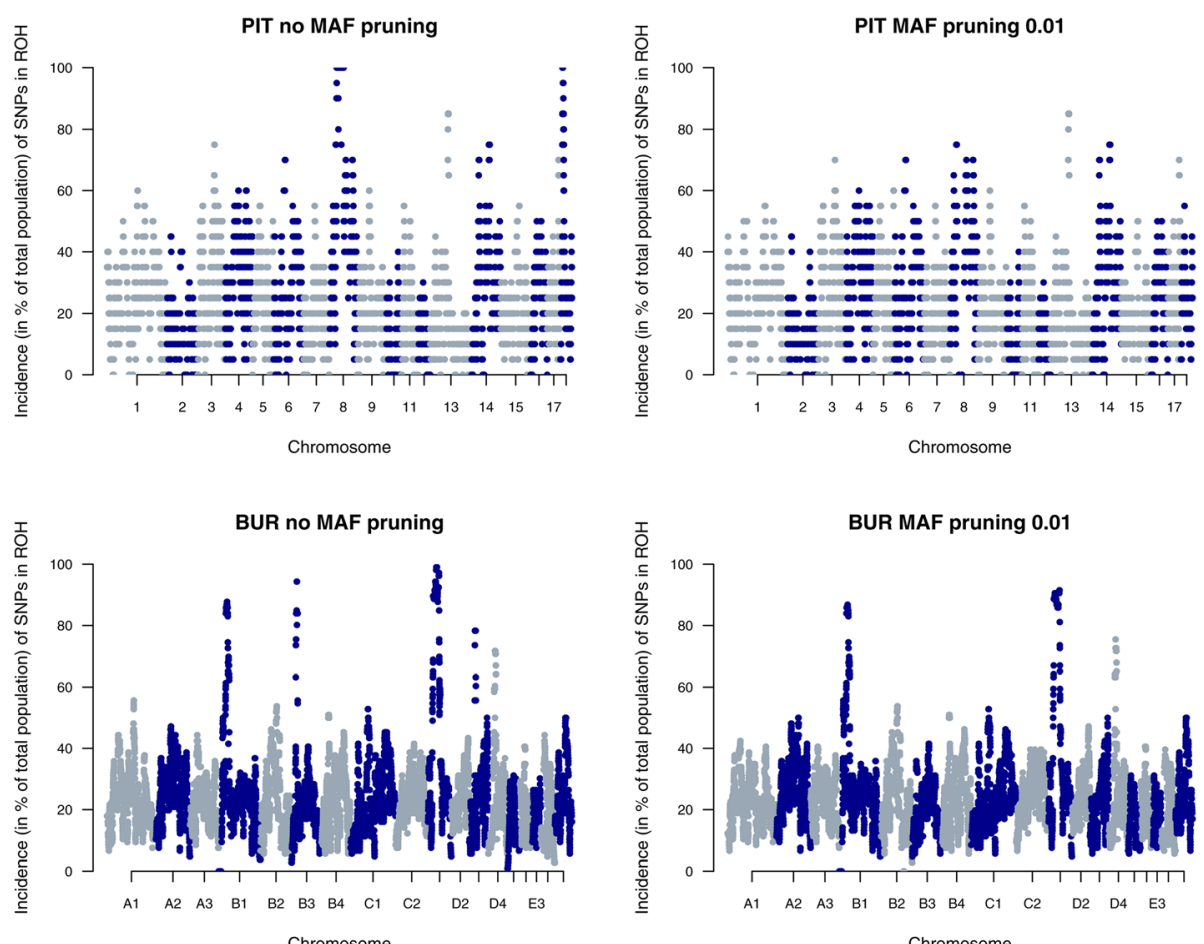

Fig. 2 Incidence plots of SNPs in a ROH for Pietrain pigs (PIT) and Burmese cats (BUR) in PLINK. Results show that pruning data for MAF (0.01) may result in a decreased $\mathrm{ROH}$ detection, especially in highly homozygous regions (e.g. SSC8 and SSC18 in PIT)

\section{Minimal density requirement}

Figure 3 presents the genome coverage (in \%) and the esti-

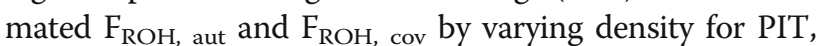
BB, MER and BUR (results for SAA, ICE, LAB and BAR are shown in Additional file 3: Figure S2). All investigated populations showed a low genome coverage with density below $40 \mathrm{~kb} / \mathrm{SNP}$. Starting from a mean density of $40 \mathrm{~kb} /$ SNP genome coverage increased and maximal coverage is reached between 60 and $75 \mathrm{~kb} / \mathrm{SNP}$.

\section{Maximal gap requirement}

The results for varying maximal gap settings in $\mathrm{ROH}$ analyses for PIT, BB, MER and BUR are shown in Fig. 4, results for SAA, ICE, LAB and BAR are added in Additional file 4: Figure S3. All investigated populations reached maximal genome coverage using gap sizes around $500 \mathrm{~kb}$. Below $500 \mathrm{~kb}$, genome coverage decreased as well as $\mathrm{F}_{\mathrm{ROH}}$ cov estimates. In general, $\mathrm{F}_{\mathrm{ROH}}$ aut decreased faster than $\mathrm{F}_{\mathrm{ROH} \text { cov}}$.

\section{Scanning window size and threshold}

An increasing scanning window size led to a decrease in estimated $\mathrm{F}_{\mathrm{ROH}}$, where especially short $\mathrm{ROH}$ were no longer detected. Similarly, an increasing threshold resulted in a decreasing $\mathrm{F}_{\mathrm{ROH}}$. For both settings, genome coverage did not vary. Results are shown in
Additional file 5: Figure S4 and Additional file 6: Figure S5.

\section{Validation using a model based approach for $\mathrm{ROH}$ detection}

In general, the model based approach (RZooRoH) yielded higher $\mathrm{F}_{\mathrm{ROH}}$ estimates than the rule based approach (PLINK) (Fig. 1 vs Fig. 5). This can be attributed to the less stringent constraints of the model based approach (e.g. no minimal $\mathrm{ROH}$ length). Pearson correlations of individual $\mathrm{F}_{\mathrm{ROH}}$ between PLINK and RZooRoH were high ( $r=0.89-0.99$ ) for all populations (no LD nor MAF pruning performed).

Results for varying LD levels prior to $\mathrm{ROH}$ analysis using a model based approach (RZooRoH) for PIT, BB, MER and BUR are shown in Fig. 5, while results for SAA, ICE, LAB and BAR are added in Additional file 7: Figure S6. MAF pruning using RZooRoH revealed the same results: in PIT and BUR, the same effects of even mild MAF pruning (0.01) on $\mathrm{ROH}$ detection were observed (Fig. 2 vs Fig. 6), whereas in the other six populations no substantial differences were apparent.

\section{Discussion}

To unravel the effects of PLINK parameter settings on $\mathrm{ROH}$ estimation using medium density SNP data we analyzed these settings on eight different livestock and pet 


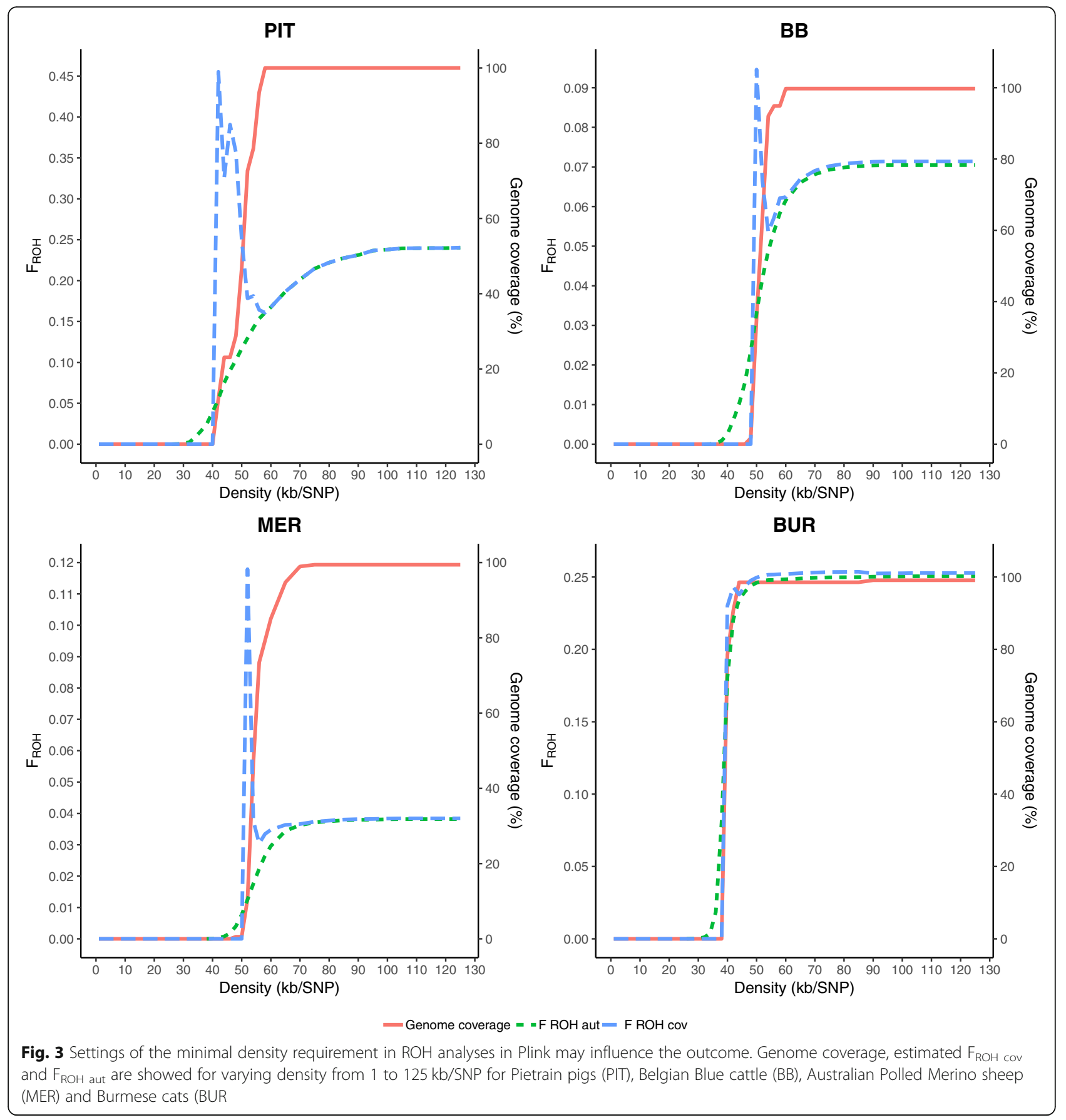

species. We examined the effects of pruning for LD and/ or MAF on $\mathrm{ROH}$ detection and genome coverage. Next, we investigated the effect of the previously unstudied PLINK parameters.

\section{Pruning for linkage disequilibrium}

The effect of LD pruning on $\mathrm{ROH}$ analysis is highly population dependent (Fig. 1 and Additional file 2: Figure S1). For PIT, genome coverage quickly drops with an increased level of LD pruning (e.g. for $R^{2}>0.5$, genome coverage is only 16.3\%). For PIT and BUR, $\mathrm{F}_{\mathrm{ROH}}$ shows a strong decrease for more stringent LD conditions, whereas in BB, MER and SAA this strong decrease could not be observed.

Howrigan et al. suggested to perform LD pruning before $\mathrm{ROH}$ analysis, based on their study using simulated genotypes [35]. However, we showed that LD pruning can have major effects on the $\mathrm{ROH}$ analysis when working with real genotypes from sampled populations. The main reason for LD pruning is to exclude short and 


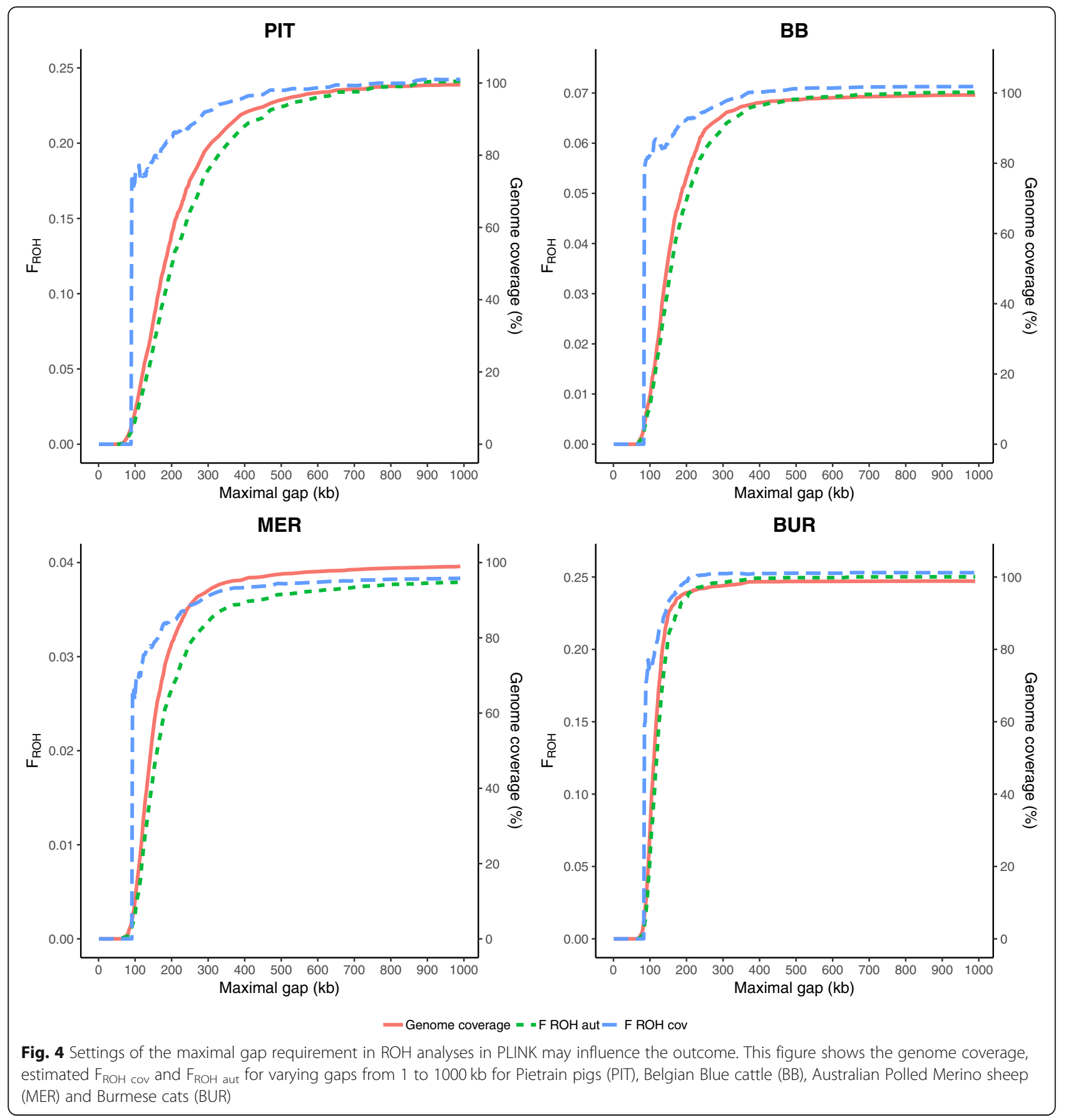

common segments that can be assigned as $\mathrm{ROH}$ but which are more likely to have arisen from LD [33, 34, 36]. However, we showed that LD pruning also affects the detection of large ROH. Furthermore, LD patterns are highly dependent on population structure [37]. In inbred populations, pruning SNP for high LD leads to a severe reduction of SNP density in homozygous genomic regions, leading to a biased $\mathrm{ROH}$ analysis. Validation using a model based approach confirmed that LD pruning has a population dependent effect on $\mathrm{ROH}$ detection
(Fig. 5 and Additional file 7: Figure S6). For BAR, at unrealistically high LD pruning levels $\left(R^{2}>0.35\right)$, deviant results were observed possibly due to the high inbreeding level, presence of microsomes and the small chicken genome.

Therefore, we argue that caution has to be taken in performing LD pruning prior to $\mathrm{ROH}$ analysis, due to differences in population structure. We suggest to correct for false positive $\mathrm{ROH}$, caused by LD, by increasing the stringency criteria for $\mathrm{ROH}$ detection (e.g. minimal 
PIT
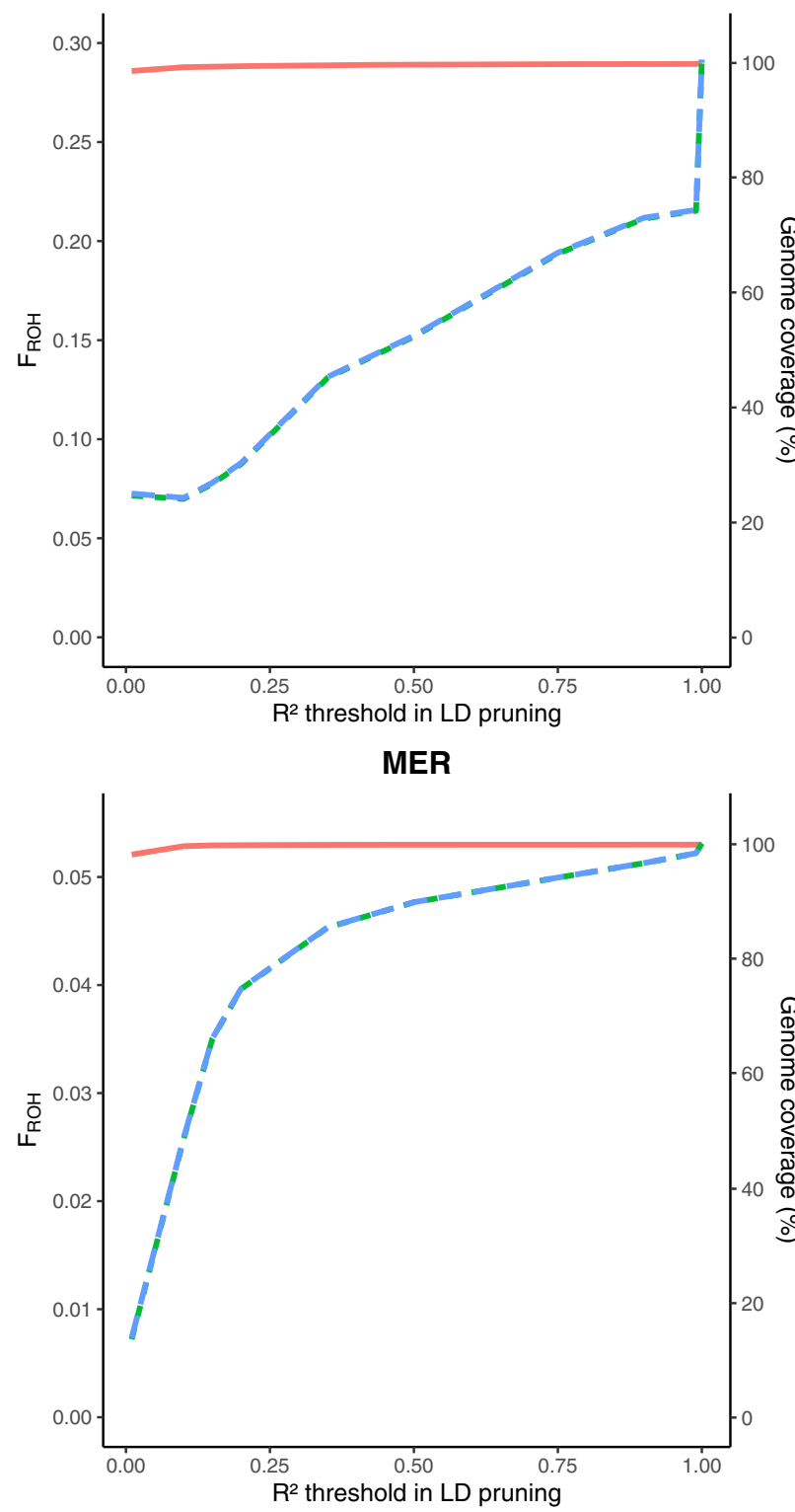

BB

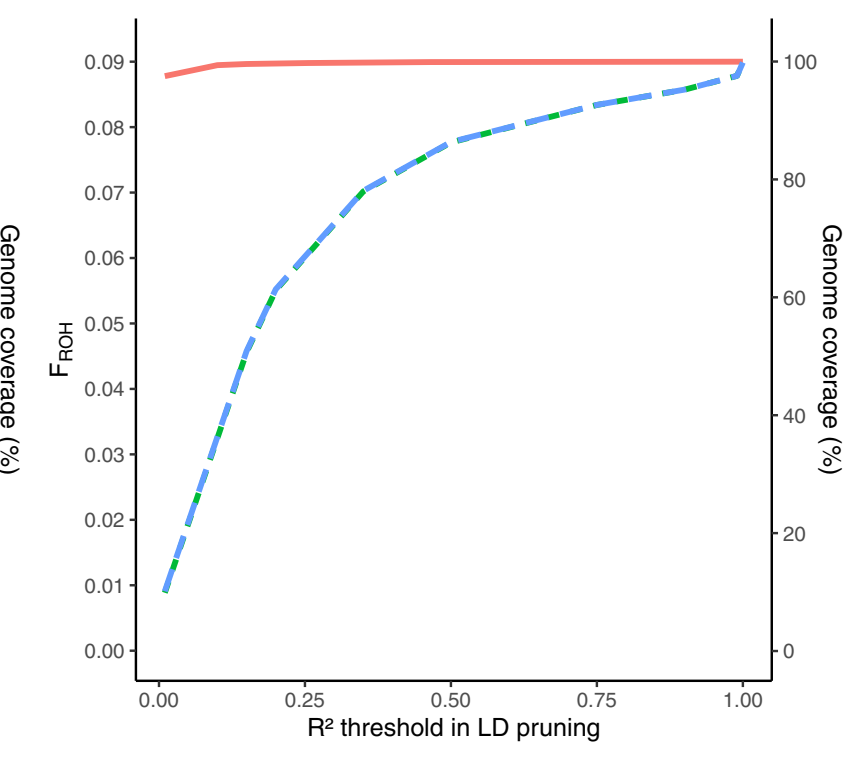

BUR

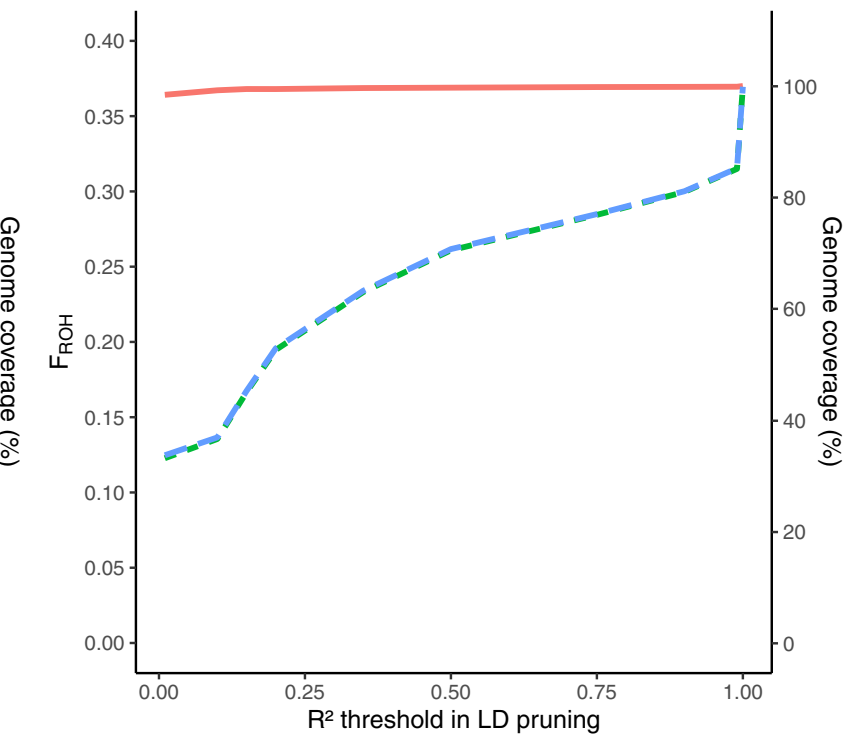

- Genome coverage - - F ROH aut - F ROH cov

Fig. 5 Pruning for linkage disequilibrium (LD) has an impact on genome coverage and FROH estimation in RZooRoH. Genome coverage and estimated $\mathrm{F}_{\mathrm{ROH}}$ cov and $\mathrm{F}_{\mathrm{ROH}}$ aut are shown for different levels of LD pruning in Pietrain pigs (PIT), Belgian Blue cattle (BB), Australian Polled Merino sheep (MER) and Burmese cats (BUR). $R^{2}$ values show the threshold values at which pruning was performed (SNPs with $R^{2}>$ threshold were pruned from the dataset). $R^{2}$ equals 1 when not pruned for LD

$\mathrm{ROH}$ length in number of SNP and kb) instead of pruning SNPs for LD, as previously reported [33, 34].

\section{Pruning for minor allele frequency}

In literature, there is no consensus whether or not genotypes should be pruned for low MAF before $\mathrm{ROH}$ analysis. Our inventory of recent studies in livestock (Table 1) showed that most studies prune data for MAF $<0.01$ or $<0.05$. Howrigan et al. [35] recommended to prune genotypes for low MAF based on their study using simulated genotypes. However, for both methods (PLINK and RZooRoH) we showed that pruning for low MAF can ignore large homozygous regions in the genome (e.g. in PIT and BUR) (Figs. 2 and 6). For PIT, MAF pruning of 0.01 overlooked large homozygous regions on SSC8 (length \pm $50 \mathrm{Mb}$ ) and on SSC18 (length $\pm 10 \mathrm{Mb}$ ). These regions also appeared nearly fixed in a study on 1632 

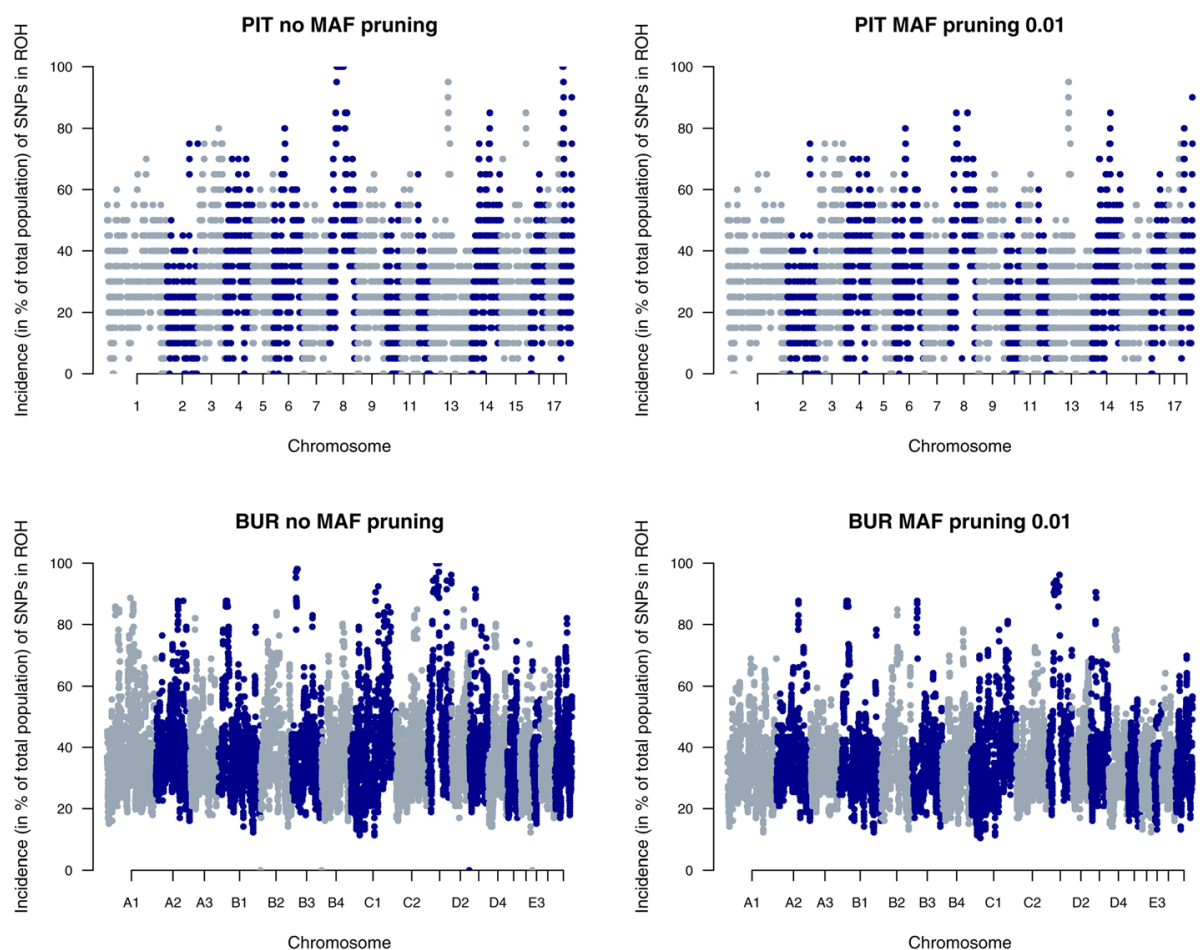

Fig. 6 Incidence plots of SNPs in a ROH for Pietrain pigs (PIT) and Burmese cats (BUR) in RZooRoH. Results show that pruning data for MAF (0.01) may result in a decreased $\mathrm{ROH}$ detection, especially in highly homozygous regions (e.g. SSC8 and SSC18 in PIT)

Pietrains [30]. For BUR, ROH went undetected on e.g. B3, D1 and D3 due to pruning for MAF. Not only $\mathrm{ROH}$ detection on fixed regions was affected by MAF pruning (e.g. D3 in BUR), but also in non-fixed regions (e.g on. B3 and D3 in BUR) large differences in $\mathrm{ROH}$ incidence were detected.

Pruning for low MAF as a quality control measure was introduced in genome wide association studies (GWAS) for two main reasons. First, genotype accuracy declines with decreasing MAF [38]. Second, the detection probability for an association decreases with lower MAF, making SNPs with low MAF less important for GWAS [38]. However, in contrast to GWAS, ROH analyses do not aim to find an association between individual SNPs and phenotype, but examine homozygous stretches of multiple SNPs. Since multiple SNPs in a sequence are evaluated, a decreased genotyping accuracy for a single SNP will have a minimal effect on the $\mathrm{ROH}$ analysis. Moreover, MAF pruning did not affect $\mathrm{F}_{\mathrm{ROH}}$ estimates and genome coverage in other populations (e.g. BB), indicating that MAF pruning does not improve $\mathrm{ROH}$ detection. Therefore, we recommend not to perform MAF pruning before $\mathrm{ROH}$ analysis.

\section{Minimal density requirement}

We showed that the minimal density setting (in $\mathrm{kb} / \mathrm{SNP}$ ) can strongly affect the outcome of the $\mathrm{ROH}$ analysis.
This is most relevant for medium density SNP arrays since in PLINK the default density setting is roughly equal to the average density for most livestock and pet species $(50 \mathrm{~kb} / \mathrm{SNP})$. This is clearly visible in Fig. 3, where genome coverage and $\mathrm{F}_{\mathrm{ROH}}$ sharply increase between 40 and $60 \mathrm{~kb} / \mathrm{SNP}$. At the default PLINK setting (50 kb/SNP), genome coverage in PIT was only $47 \%$ and for MER barely $0.6 \%$. Calculating the genome coverage parameter proves to be a good method to check whether the density parameter is set appropriately. For the studied populations maximal genome coverage was reached between 60 and $70 \mathrm{~kb} / \mathrm{SNP}$.

Moreover, SNP densities can differ along the genome (e.g. $>150 \mathrm{~kb} / \mathrm{SNP}$ on SSC3 in PIT) and therefore certain regions could be overlooked. A possible solution is to implement a check for density on the scanning window level in PLINK's algorithm (implement density as --homozyg-window-density instead of --homozyg-density). This forces the algorithm to check every window for the required minimal density instead of only performing the check on (large) homozygous regions.

Note that the average chicken genome length $( \pm 1000$ $\mathrm{Mb})$ is about half the size of the average mammalian genome, and therefore the minimal density at which maximal genome coverage is reached lies around $25 \mathrm{~kb} /$ SNP (e.g. BAR, Additional file 3: Figure S2). Furthermore, LAB was genotyped on an $110 \mathrm{~K}$ array and thus 
the average SNP density was twice as dense as the other populations, causing maximal genome coverage to be reached at $\pm 20 \mathrm{~kb} / \mathrm{SNP}$ (Additional file 3: Figure S2).

\section{Maximal gap requirement}

A wide variety of gap settings is reported in literature and little motivation is given for their use (Table 1). For all studied populations, the default PLINK gap (1000 kb) covers over $99 \%$ of the detectable autosome (Fig. 4). Only for gap sizes below $500 \mathrm{~kb}$, genome coverage drops below $95 \%$. The default value of $1000 \mathrm{~kb}$ is therefore set too high to influence $\mathrm{ROH}$ detection even for medium density data. To determine the optimal gap setting, high density genotypes or sequence data are necessary. This way, one can determine at which maximal gap it is no longer safe to assume that underlying SNPs are homozygous. Anticipating further research, we suggest to minimize gap length while maintaining maximal genome coverage and we advise to use $\mathrm{F}_{\mathrm{ROH}}$ cov for inbreeding estimation.

\section{Scanning window size and threshold}

With an increasing window size and window threshold, $\mathrm{F}_{\mathrm{ROH}}$ decreases due to more stringent conditions to identify a homozygous segment. We recommend choosing the scanning window size parameter equal to $L$, the minimal length of a ROH. On the one hand, setting the scanning window size below $L$ will not result in the detection of new $\mathrm{ROH}$, as the minimal length of a $\mathrm{ROH}$ is set at $L$. On the other hand, a window size higher than $L$ SNPs prohibits the detection of $\mathrm{ROH}$ with a minimal size of $L$ SNPs, as Curik et al. also pointed out [3].

The scanning window threshold affects the number of outer SNPs in a homozygous segment that cannot be included reliably in the final ROH segment. After all, it is possible that outer SNPs of the homozygous segment are homozygous rather by chance than by descent.

We propose to calculate the scanning window threshold $(t)$ as follows:

$$
t=\text { floor }\left(\frac{N_{\text {out }}+1}{L}, 3\right)
$$

with $\mathrm{N}_{\text {out }}$ the desired number of final outer SNPs on either side of the homozygous segment that should not be included in the final $\mathrm{ROH}$ and $L$ the scanning window size. In this formula, ' +1 ' is included as this denotes the first SNP that will be tolerated of the final ROH and ', 3' points at flooring with three decimals. For example, with $L=100$ and $\mathrm{N}_{\text {out }}=4$, the threshold will be set at 0.05 . By doing this, we will scan windows of 100 SNPs and in the obtained homozygous segment we discard the four outer SNPs on each side of the homozygous segment.
Most studies do not report the scanning window threshold setting, although it impacts the outcome of $\mathrm{ROH}$ analysis. Therefore, we encourage authors to always specify the scanning window threshold.

\section{Comparison with literature}

This study shows that PLINK settings and pruning for either LD or MAF are extremely important for $\mathrm{ROH}$ analysis outcome. Nevertheless, as Table 1 shows, several parameter settings are often not explicitly mentioned. Therefore it is unclear whether some settings were left unadjusted or ignored. Ten out of 23 studies in Table 1 used a density of $50 \mathrm{~kb} / \mathrm{SNP}$ or did not mention a change of the density setting. It is possible that genome coverage in these studies is strongly reduced and consequently, $\mathrm{F}_{\mathrm{ROH}}$ could be underestimated for these studies.

As an example, we evaluated the genome coverage of the $\mathrm{ROH}$ analysis as performed by Yang et al. on 146 pig populations [24]. We found a genome coverage of only $34.2 \%$, mainly due to MAF pruning and use of the unadapted default density parameter [24]. Therefore, the $\mathrm{F}_{\mathrm{ROH}}$ estimate for PIT was equal to $7.6 \%$ (using the $\mathrm{F}_{\mathrm{ROH}}$ calculation method of McQuillan et al. [6]), whereas we estimated the average $\mathrm{F}_{\mathrm{ROH}}$ for PIT to be at least a threefold higher. However, the spearman rank correlation on population level for all 146 populations between our estimated $\mathrm{F}_{\mathrm{ROH}}$ cov and the average $\mathrm{F}_{\mathrm{ROH}}$ reported by Yang et al. was high $(r=0.90)$.

\section{Conclusions}

This study has shown that MAF and LD pruning as well as PLINK input settings can severely impact $\mathrm{ROH}$ analyses on medium density genotypes.

Pruning for low MAF and LD was historically introduced in genomic analyses but seems to provide little benefits for $\mathrm{ROH}$ studies. Our findings show that MAF and LD pruning can be problematic for $\mathrm{ROH}$ detection, regardless of the method used (rule based or model based). Therefore we recommend to skip MAF and LD pruning prior to all $\mathrm{ROH}$ analyses using medium density genotypes.

It is clear from our results that a low minimal density setting (in $\mathrm{kb} / \mathrm{SNP}$ ) can lead to an incomplete genome coverage of the analysis and should be evaluated thoroughly. Moreover, the default PLINK setting of $50 \mathrm{~kb} /$ SNP is often not suitable. Furthermore, we advise to minimize the maximal gap setting while still assuring maximal genome coverage. The scanning window size should be kept equal to the minimal desired $\mathrm{ROH}$ length. Finally, a proposal to calculate the scanning window threshold is given.

Overall, we advocate to always report PLINK input settings in publications, and to validate them prior to analysis. Moreover, we encourage to use and report 
genome coverage to reflect the $\mathrm{ROH}$ analysis' validity. Based on this genome coverage, $\mathrm{F}_{\mathrm{ROH}}$ can be more accurately estimated. We strongly believe that using these recommendations will improve quality and comparability of $\mathrm{ROH}$ analyses.

\section{Material and methods}

The effect of LD and MAF pruning on $\mathrm{ROH}$ detection, as well as the effects of the density, gap and window size settings were evaluated on different livestock and pet species (pig, sheep, horse, cattle, goat, chicken, cat and dog). We demonstrated our findings in one example population per species (Table 2).

All populations were genotyped on medium density arrays. We performed our analyses on the autosomal genome, discarding all SNPs with unassigned chromosomal information and all SNPs with a low call rate $(<$ 95\%). Detailed information on the quality control for all populations is given in Additional file 1: Table S1.

Many studies estimate the inbreeding coefficient based on $\mathrm{ROH}\left(\mathrm{F}_{\mathrm{ROH}}\right)$ as [6]:

$$
F_{R O H}=\frac{L_{R O H}}{L_{\text {aut }}},
$$

where $L_{R O H}$ is the total length of all ROHs in the individual's genome, and $L_{a u t}$ is the length of the autosomal genome. The population's mean $\mathrm{F}_{\mathrm{ROH}}$ is calculated as the average $\mathrm{F}_{\mathrm{ROH}}$ of all individuals. However, the length of the autosomal genome depends on the genome assembly used for SNP mapping and can therefore differ between genotyping arrays. Moreover, regional differences in SNP density can result in genomic regions where it is impossible to detect $\mathrm{ROH}$. The detection of $\mathrm{ROH}$ in these regions is not only dependent on the SNP density of the array, but also on the specific criteria assigned to PLINK to detect ROH.

Therefore, we propose two different methods for estimating $\mathrm{F}_{\mathrm{ROH}}$. First $\mathrm{F}_{\mathrm{ROH}}$, aut is the estimated degree of inbreeding based on the length of the autosomal genome, with $L_{\text {aut }}$ calculated as the length between the first SNP and the last SNP per chromosome for all autosomal chromosomes. Second, $\mathrm{F}_{\mathrm{ROH}}$ cov is the estimated degree of inbreeding based on the length of the covered genome, where $L_{\text {aut }}$ is equal to the length of the autosomal genome where $\mathrm{ROH}$ detection is possible. This was calculated by simulating an individual with a completely homozygous genotype (based on the population's .map file) and performing the $\mathrm{ROH}$ analysis with all specified parameters on this homozygous individual. The total $\mathrm{ROH}$ length found for this homozygous individual is the maximal detectable $\mathrm{ROH}$ length for any individual in this population, given the parameter settings. Similarly, we calculated the genome coverage of the $\mathrm{ROH}$ analysis
Table 2 Overview of the selected populations per species

\begin{tabular}{lllll}
\hline Species & Population & Abbreviation & N & Source \\
\hline Pig & Pietrain & PIT & 20 & {$[24]$} \\
Cattle & Belgian Blue & BB & 766 & Own data \\
Sheep & Australian Polled Merino & MER & 98 & {$[39]$} \\
Horse & Swedish bred Icelandic & ICE & 209 & {$[40]$} \\
Goat & Saanen & SAA & 171 & {$[41]$} \\
Chicken & Barnevelder & BAR & 24 & {$[25]$} \\
Cat & Burmese & BUR & 106 & {$[42]$} \\
Dog & Labrador & LAB & 728 & {$[43]$} \\
\hline
\end{tabular}

as the proportion of the maximal detectable $\mathrm{ROH}$ length over the length of the (autosomal) genome. This genome coverage was as an indication of the validity of the $\mathrm{ROH}$ analysis.

To study the effect of MAF pruning, we used PLINK's --maf function for MAF equal to 0.01, 0.05, 0.10 and 0.20 and compared this to the $\mathrm{ROH}$ analysis without MAF pruning. To analyze the effect of LD pruning on $\mathrm{ROH}$ analyses, we used PLINK's --indep-pairwise function with a scanning window of 50 (step size of 5) and pruned SNPs with $R^{2}$ values between 0.01 and 0.99 . These results were compared to the $\mathrm{ROH}$ analysis without LD pruning $\left(R^{2}=1\right)$. To test the minimal density setting, we varied --homozyg-density from 10 to $125 \mathrm{~kb} / \mathrm{SNP}$. To examine the effect of the maximal gap setting, we varied --homozyg-gap from 1 to $1000 \mathrm{~kb}$. The scanning window size setting (--homozyg-window-snp) was investigated by varying this setting from 1 to 150 SNPs. The scanning window threshold (--homozyg-window-threshold) was varied between 0.05 and 0.95 . The PLINK settings were evaluated on non-pruned genotypes. When unvaried, $\mathrm{ROH}$ detection settings were set to a small scanning window (20 SNPs), a large gap (2 Mb), a high density level $(200 \mathrm{~kb} / \mathrm{SNP})$ and a scanning window threshold level of 0.05. All $\mathrm{ROH}$ detection was performed with a minimal $\mathrm{ROH}$ length of $1 \mathrm{Mb}$, maximum one missing SNP and no heterozygous SNPs in the scanning window. The minimal number of SNPs in a ROH was determined by the formula proposed by Lencz et al. and adapted by Purfield et al. [32, 33]:

$$
L=\frac{\log _{e} \frac{\alpha}{n_{s} n_{i}}}{\log _{e}(1-h e t)},
$$

with $n_{s}$ the number of genotyped SNPs per individual, $n_{i}$ the number of genotyped individuals, $\alpha$ the percentage of false positive $\mathrm{ROH}(0.05)$ and het the mean heterozygosity across all SNPs. 
We validated our results using PLINK with a non-rule based $\mathrm{ROH}$ detection method by analyzing all populations using the model based software RZooRoH, developed by Druet and Gauthier [44]. The RZooRoH software identifies homozygous-by-descent (HBD) segments associated with $\mathrm{ROHs}$ and is based on a hidden Markov model framework. We used a two-states model (1R model), which estimates the probability between two consecutive markers to be either HBD or non-HBD [44]. The genotyping error rate was set to $0.25 \%$, as suggested by Ferenčaković et al. [34]. Furthermore, allele frequencies of reference populations were provided to the algorithm, as suggested by Dr. Tom Druet (personal communication). To compare $\mathrm{F}_{\mathrm{ROH}}$ results from both PLINK and RZooRoH, an individual with a completely homozygous genotype was simulated, using the same method as previously described. This individual was analyzed in RZooRoH to calculate the maximal detectable $\mathrm{ROH}$ length for any individual in the population, given the model settings.

\section{Supplementary information}

Supplementary information accompanies this paper at https://doi.org/10. 1186/s12864-020-6463-X.

Additional file 1: Table S1. Quality control metrics for all evaluated populations. Abbreviations as in Table 2.

Additional file 2: Figure S1. The effect of linkage disequilibrium (LD) pruning on genome coverage and $\mathrm{F}_{\mathrm{ROH}}$ estimates for ICE, SAA, LAB and BAR in PLINK.

Additional file 3: Figure S2. The effect of the density setting (in kb/ SNP) on genome coverage and $F_{R O H}$ estimates for ICE, SAA, LAB and BAR.

Additional file 4: Figure $\mathbf{S 3}$. The effect of maximal gap setting (in kb) on genome coverage and $F_{\mathrm{ROH}}$ estimates for ICE, SAA, LAB and BAR.

Additional file 5: Figure S4. The effect the scanning window size on genome coverage and $\mathrm{F}_{\mathrm{ROH}}$ estimates for all evaluated populations.

Additional file 6: Figure S5. The effect of the scanning window threshold on genome coverage and $\mathrm{F}_{\mathrm{ROH}}$ estimates for all evaluated populations.

Additional file 7: Figure S6. The effect of linkage disequilibrium (LD) pruning on genome coverage and $\mathrm{F}_{\mathrm{ROH}}$ estimates for ICE, SAA, LAB and $\mathrm{BAR}$ in RZooRoH. For BAR, an increase in $\mathrm{F}_{\mathrm{ROH}}$ was detected at very low $R^{2}$ values (> 0.35), probably linked to a high degree of inbreeding and a strong decrease in number of markers in a small genome to reliably estimate $\mathrm{HBD}$.

\section{Abbreviations}

BAR: Barnevelder chicken population; BB: Belgian Blue cattle population; BUR: Burmese cat population; $\mathrm{F}_{\mathrm{ROH}}$ aut: Genomic $\mathrm{ROH}$ inbreeding coefficient (calculation based on autosomal genome length); $\mathrm{F}_{\mathrm{ROH} \text { cov: Genomic } \mathrm{ROH}}$ inbreeding coefficient (calculation based on the genome coverage); $\mathrm{F}_{\mathrm{ROH}}$ : Genomic inbreeding coefficient based on ROH; GWAS: Genome wide association study; HBD: Homozygous-by-descent; het: Mean heterozygosity across all SNPs; ICE: Swedish bred Icelandic horse population; kb: Kilo base pairs; L: Minimal required ROH length in SNPs; LAB: Labrador dog population; $L_{\text {aut }}$ : Length of the autosomal genome; LD: Linkage disequilibrium; $\mathrm{L}_{\mathrm{ROH}}$ : Length of all ROHs in an individual's genome; MAF: Minor allele frequency; Mb: Mega base pairs; MER: Australian polled Merino sheep population; $n_{i}$ : Number of genotyped individuals; $N_{\text {out }}$ : Desired number of final outer SNPs on either side of the homozygous segment that should not be included in the final $\mathrm{ROH} ; \mathrm{n}_{\mathrm{s}}$ : Number of genotyped SNPs per individual;
PIT: Pietrain pig population; $\mathrm{R}^{2}$ : Inter-variant allele count squared correlations; $\mathrm{ROH}$ : Run of homozygosity; SAA: Saanen goat population; SNP: Single nucleotide polymorphism; t: Threshold of PLINK's scanning window; a: Percentage of false positive $\mathrm{ROH}(0.05)$

\section{Acknowledgements}

The authors would like to acknowledge the suggestions and clarifications from Dr. Christopher Chang regarding the PLINK ROH detection algorithm. Furthermore, we would like to thank Dr. Tom Druet for his advice on the use of the RZooRoH algorithm.

\section{Authors' contributions}

RM and WG analyzed the data and wrote the manuscript. RM, WG, SJ and NB designed and conceived this study. SJ and NB critically reviewed the analyses and the manuscript. All authors read and approved the final manuscript.

\section{Funding}

This study was partially funded by an SB PhD fellowship (1S37119N) and an FR PhD fellowship (1104320 N) of the Research Foundation Flanders (FWO). Belgian Blue genotypes were generated in projects supported by the Belgian Federal Public Service of Health, Food Chain Safety and Environment (RT11/5 and RT17/1). The funding bodies played no role in the design of the study and collection, analysis, and interpretation of data and in writing the manuscript.

\section{Availability of data and materials}

The dataset of Belgian Blue genotypes used in the current study are accessible via Figshare [DOI: https://doi.org/10.6084/m9.figshare.11441283].

The online datasets are available at:

- Bortoluzzi C, Crooijmans RPMA, Bosse M, Hiemstra SJ, Groenen MAM, Megens H (2018) Data from: The effects of recent changes in breeding preferences on maintaining traditional Dutch chicken genomic diversity. Dryad Digital Repository. https://doi.org/10.5061/dryad.1d832h3

- Colli L, Milanesi M, Talenti A, Bertolini F, Chen M, Crisà A, Daly K, Del Corvo M, Guldbrandtsen B, Lenstra JA, Rosen BD, Vajana E, Catillo G, Joost S, Nicolazzi EL, Rochat E, Rothschild MF, Servin B, Sonstegard TS, Steri R, Van Tassel CP, Ajmone-Marsan P, Crepaldi P, Stella A, AdaptMap Consortium (2018) Data from: Signatures of selection and environmental adaptation across the goat genome post-domestication. Dryad Digital Repository. https://doi.org/10.5061/dryad.v8g21pt

- Gandolfi, B., Alhaddad, H., Abdi, M., Bach, L. H., Creighton, E. K., Davis, B. W.,

.. Lyons, L. A. (2018). Applications and efficiencies of the first cat $63 \mathrm{~K}$ DNA array. Scientific Reports, 8(1), 7024. Data available on: https://www.nature.com/ articles/s41598-018-25438-0\#Sec26

- Kijas, J. W., Lenstra, J. A., Hayes, B., Boitard, S., \& Neto, P. (2012). GenomeWide Analysis of the World's Sheep Breeds Reveals High Levels of Historic Mixture and Strong Recent Selection. PLoS Biology, 10(2), 1001258. Data available on: http://www.sheephapmap.org/download.php

- Shrestha M, Eriksson S, Schurink A, Andersson LS, Sundquist M, Frey R, Broström H, Bergström T, Ducro B, Lindgren G (2015) Data from: Genomewide association study of insect bite hypersensitivity in Swedish-born Icelandic horses. Dryad Digital Repository. https://doi.org/10.5061/dryad.9r161.2 - Wiener P, Sánchez-Molano E, Clements DN, Woolliams JA, Haskell MJ, Blott SC (2017) Data from: Genomic data illuminates demography, genetic structure and selection of a popular dog breed. Dryad Digital Repository. https://doi.org/10.5061/dryad.38q43

- Yang B, Cui L, Perez-Enciso M, Traspov A, Crooijmans RPMA, Zinovieva N, Schook LB, Archibald A, Gatphayak K, Knorr C, Triantafyllidis A, Alexandri P, Semiadi G, Hanotte O, Dias D, Dovč P, Uimari P, lacolina L, Scandura M, Groenen MAM, Huang L, Megens H (2017) Data from: Genome-wide SNP data unveils the globalization of domesticated pigs. Dryad Digital Repository. https:// doi.org/10.5061/dryad.30tk6

Ethics approval and consent to participate Not applicable. 


\section{Competing interests}

The authors declare that they have no competing interests.

\section{Received: 17 May 2019 Accepted: 8 January 2020}

\section{10 .}

\section{References}

1. Peripolli E, Munari DP, Silva MVGB, Lima ALF, Irgang R, Baldi F. Runs of homozygosity: current knowledge and applications in livestock. Anim Genet. 2017;48(3):255-71 Available from: http://doi.wiley.com/10.1111/ age.12526. Cited 29 Jun 2018.

2. Ceballos FC, Joshi PK, Clark DW, Ramsay M, Wilson JF. Runs of homozygosity: windows into population history and trait architecture. Nat Publ Gr. 2018;19 Available from: www.nature.com/nrg. Cited 26 Sept 2018.

3. Curik I, Ferenčaković M, Sölkner J. Inbreeding and runs of homozygosity: a possible solution to an old problem. Livest Sci. 2014;166:26-34. https://doi org/10.1016/j.livsci.2014.05.034 Cited 26 Sept 2018.

4. Broman KW, Weber JL. Long homozygous chromosomal segments in reference families from the centre d'Etude du polymorphisme humain. Am J Hum Genet. 1999;65 Available from: https://www.ncbi.nlm.nih.gov/pmc/ articles/PMC1288359/pdf/AJHGv65p1493.pdf. Cited 17 Dec 2018.

5. Gibson J, Morton NE, Collins A. Extended tracts of homozygosity in outbred human populations. Hum Mol Genet. 2006;15(5):789-95 Available from: https://academic.oup.com/hmg/article-abstract/15/5/789/596723. Cited 17 Dec 2018.

6. Mcquillan R, Leutenegger A-L, Abdel-Rahman R, Franklin CS, Pericic M, Barac-Lauc L, et al. Runs of homozygosity in European populations. Am J Hum Genet. 2008;83(3):359-72 Available from: https://ac.els-cdn.com/S0002 92970800445X/1-s2.0-S000292970800445X-main.pdf?_tid=a5fd94b2-82f8-4b5 e-844d-b7f750c63470\&acdnat=1545042424_f3ed794da5792026f6687a7b53 a4a351. Cited 17 Dec 2018.

7. Purcell $S$, Neale B, Todd-Brown K, Thomas L, Ferreira MAR, Bender D, et al. PLINK: a tool set for whole-genome association and population-based linkage analyses. Am J Hum Genet Am J Hum Genet. 2007;8181:559-75 Available from: www.ajhg.org. Cited 10 Aug 2017.

8. Chang CC, Chow CC, Tellier LC, Vattikuti S, Purcell SM, Lee JJ. Secondgeneration PLINK: rising to the challenge of larger and richer datasets. Gigascience. 2015;4(7):1-16 Available from: https://academic.oup.com/ gigascience/article-abstract/4/1/1/2707533. Cited 20 July 2018.

9. Bosse $M$, Megens $\mathrm{H}-\mathrm{J}$, Madsen $\mathrm{O}$, Paudel $Y$, Frantz LA, Schook LB, et al. Regions of homozygosity in the porcine genome: consequence of demography and the recombination landscape. PLoS Genet. 2012;8(11): 1003100 Available from: www.plosgenetics.org. Cited 11 Oct 2018.

10. Ai H, Huang L, Ren J. Genetic diversity, linkage disequilibrium and selection signatures in Chinese and Western pigs revealed by genome-wide SNP markers. PLoS One. 2013:8(2):e56001 Kozak CA, editor. Available from: https://dx.plos.org/10.1371/journal.pone.0056001. Cited 3 May 2019.

11. Bjelland DW, Weigel KA, Vukasinovic N, Nkrumah JD. Evaluation of inbreeding depression in Holstein cattle using whole-genome SNP markers and alternative measures of genomic inbreeding. J Dairy Sci. 2013;96(7): 4697-706 Available from: https://www.sciencedirect.com/science/article/pii/ S0022030213003706?via\%3Dihub. Cited 3 May 2019.

12. Manuel Herrero-Medrano J, Megens H-J, Am Groenen M, Ramis G, Bosse M, Pérez-Enciso M, et al. Conservation genomic analysis of domestic and wild pig populations from the Iberian Peninsula. 2013. Available from: http://www.biomedcentral.com/1471-2156/14/106. Cited 26 Sept 2018

13. Biscarini F, Biffani S, Nicolazzi EL, Morandi N, Stella A. Applying runs of homozygosity to the detection of associations between genotype and phenotype in farm animals. In Proceedings of the 10th World Congress on Genetics Applied to Livestock Production, Vol. 675. 2014. pp. 1-3.

14. Scraggs E, Zanella R, Wojtowicz A, Taylor JF, Gaskins CT, Reeves JJ, et al. Estimation of inbreeding and effective population size of full-blood wagyu cattle registered with the American Wagyu Cattle Association. J Anim Breed Genet. 2014;131(1):3-10 Available from: http://doi.wiley.com/10.1111/jbg.12 066. Cited 3 May 2019

15. Zhang Y, Young JM, Wang C, Sun X, Wolc A, Dekkers JCM. Inbreeding by pedigree and genomic markers in selection lines of pigs. In: Proceedings, 10 th world congress of genetics applied to livestock production inbreeding by pedigree and genomic markers in selection lines of pigs. Vancouver; 2010. Available from: http://www.wcgalp.org/system/files/proceedings/2 014/inbreeding-pedigree-and-genomic-markers-selection-lines-pigs.pdf. Cited 3 May 2019.

16. Al-Mamun HA, Clark SA, Kwan P, Gondro C. Genome-wide linkage disequilibrium and genetic diversity in five populations of Australian domestic sheep. Genet Sel Evol. 2015;47:90 Available from: http://www. polldorset.org.au. Cited 17 Aug 2018.

17. Mészáros G, Stückler MP, Ferenčaković M, Sölkner J. Genomic background of entropion in Fleckvieh cattle. Poljoprivreda. 2015;21(1 SUPPLEMENT):48-51.

18. Muchadeyi FC, Malesa MT, Soma P, Dzomba EF. Runs of homozygosity in Swakara pelt producing sheep: implications on sub-vital performance. In Proc. Assoc. Advmt. Anim. Breed. Genet, Vol. 21. 2015. pp. 310-13.

19. Rodríguez-Ramilo ST, Fernández J, Toro MA, Hernández D, Villanueva B. Genome-wide estimates of coancestry, inbreeding and effective population size in the Spanish Holstein population. PLoS One. 2015;10(4):e0124157 Hansen PJ, editor. Available from: https://dx.plos.org/10.1371/journal.pone. 0124157. Cited 3 May 2019.

20. Zhang Q, Calus MP, Guldbrandtsen B, Lund MS, Sahana G. Estimation of inbreeding using pedigree, 50k SNP chip genotypes and full sequence data in three cattle breeds. BMC Genet. 2015;16:88 Available from: http://www.rproject.org/. Cited 17 Aug 2018.

21. Zanella R, Peixoto JO, Cardoso FF, Cardoso LL, Biegelmeyer P, Cantão ME, et al. Genetic diversity analysis of two commercial breeds of pigs using genomic and pedigree data. Genet Sel Evol. 2016;48:24 Available from: http://www.animalgenome.org/repository/. Cited 3 May 2019.

22. François L, Wijnrocx K, Colinet G, Gengler N, Hulsegge B, Windig JJ, et al. Genomics of a revived breed: case study of the Belgian campine cattle. PLoS One. 2017;12(4). https://doi.org/10.1371/journal.pone.0175916.t001 Cited 26 Sept 2018.

23. Purfield DC, Mcparland S, Wall E, Berry DP. The distribution of runs of homozygosity and selection signatures in six commercial meat sheep breeds. PLoS One. 2017;12(5) Available from: http://journals.plos.org/plosone/article/ file?id=10.1371/journal.pone.0176780\&type=printable. Cited 11 Jun 2018.

24. Yang B, Cui L, Perez-Enciso M, Traspov A, Crooijmans RP, Zinovieva N, et al. Genome-wide SNP data unveils the globalization of domesticated pigs. Genet Sel Evol. 2017:49:71 Available from: www.fao.org. Cited 29 Mar 2019.

25. Bortoluzzi C, Crooijmans RPMA, Bosse M, Hiemstra SJ, Groenen MAM, Megens $\mathrm{H}$-J. The effects of recent changes in breeding preferences on maintaining traditional Dutch chicken genomic diversity. Heredity (Edinb). 2018;121(6):564-78 Available from: http://www.nature.com/articles/s41437018-0072-3. Cited 23 Apr 2019

26. Kumar C, Song S, Dewani P, Kumar M, Parkash O, Ma Y, Jiang L. Population structure, genetic diversity and selection signatures within seven indigenous Pakistani goat populations. Animal genetics. 2018;49(6):592-604.

27. Mastrangelo S, Ciani E, Sardina MT, Sottile G, Pilla F, Portolano B. Runs of homozygosity reveal genome-wide autozygosity in Italian sheep breeds. Anim Genet. 2018;49(1):71-81 Available from: http://doi.wiley.com/10.1111/ age.12634. Cited 26 Sept 2018.

28. Michailidou S, Tsangaris G, Fthenakis GC, Tzora A, Skoufos I, Karkabounas SC, et al. Genomic diversity and population structure of three autochthonous Greek sheep breeds assessed with genome-wide DNA arrays. Mol Genet Genomics. 2018;293:753-68. https://doi.org/10.1007/s00438-018-1421-x Cited 17 Aug 2018.

29. Zhang Z, Zhang Q, Xiao Q, Sun H, Gao H, Yang Y, et al. Distribution of runs of homozygosity in Chinese and Western pig breeds evaluated by reducedrepresentation sequencing data. Anim Genet. 2018;49(6):579-91 Available from: http://doi.wiley.com/10.1111/age.12730. Cited 2 Apr 2019.

30. Gorssen W, Meyermans R, Buys N, Janssens S. SNP genotypes reveal breed substructure, selection signatures and highly inbred regions in Piétrain pigs. Anim Genet. 2020;51:32-42. https://doi.org/10.1111/age.12888.

31. Meyermans R, Gorssen W, Wijnrocx K, Lenstra JA, Vellema P, Buys N, Janssens S. Unraveling the genetic diversity of Belgian Milk Sheep using medium-density SNP genotypes. Anim Genet. 2020. https://doi.org/10.1111/ age.12891.

32. Lencz T, Lambert C, DeRosse P, Burdick KE, Morgan TV, Kane JM, et al. Runs of homozygosity reveal highly penetrant recessive loci in schizophrenia. Proc Natl Acad Sci U S A. 2007;104(50):19942-7 Available from: http://www. ncbi.n/m.nih.gov/pubmed/18077426. Cited 3 May 2019.

33. Purfield DC, Berry DP, Mcparland S, Bradley DG. Runs of homozygosity and population history in cattle. BMC Genet. 2012;13:70 Available from: http:// www.biomedcentral.com/1471-2156/13/70. Cited 17 Aug 2018. 
34. Ferenčaković M, Sölkner J, Curik I. Estimating autozygosity from highthroughput information: effects of SNP density and genotyping errors, Genet Sel Evol. 2013;45:42 Available from: www.goldenhelix.com. Cited 17 Aug 2018.

35. Howrigan DP, Simonson MA, Keller MC. Detecting autozygosity through runs of homozygosity: a comparison of three autozygosity detection algorithms. BMC Genomics. 2011;12(1):460 Available from: http:// bmcgenomics.biomedcentral.com/articles/10.1186/1471-2164-12-460. Cited 3 May 2019

36. Kirin M, McQuillan R, Franklin CS, Campbell H, McKeigue PM, Wilson JF. Genomic runs of homozygosity record population history and consanguinity. PLoS One. 2010;5(11):e13996 Kayser M, editor. Available from: http://dx.plos.org/10.1371/journal.pone.0013996. Cited 25 May 2018.

37. Hartl DL, Clark AG. Principles of population genetics. 3rd ed. Sunderland: Sinauer Associates, Inc.; 1997. p. 104-5.

38. Weale ME. Quality control for genome-wide association studies. In: Barnes MR, Breen G, editors. Genetic variation. Totowa: Humana Press; 2010. p. 341-72. Available from: http://link.springer.com/10.1007/978-1-60327-36 7-1_19. Cited 3 May 2019.

39. Kijas JW, Lenstra JA, Hayes B, Boitard S, Neto P. Genome-wide analysis of the world's sheep breeds reveals high levels of historic mixture and strong recent selection. PLoS Biol. 2012:10(2):1001258 Available from: www. plosbiology.org. Cited 10 Jan 2019.

40. Shrestha M, Eriksson S, Schurink A, Andersson LS, Sundquist M, Frey R, et al. Genome-wide association study of insect bite hypersensitivity in Swedishborn Icelandic horses. J Hered. 2015;106(4):366-74 Available from: https:// academic.oup.com/jhered/article-lookup/doi/10.1093/jhered/esv033. Cited 3 May 2019.

41. Colli L, Milanesi M, Talenti A, Bertolini F, Chen M, Crisà A, et al. Genomewide SNP profiling of worldwide goat populations reveals strong partitioning of diversity and highlights post-domestication migration routes. Genet Sel Evol. 2018;50(1):58 Available from: https://gsejournal. biomedcentral.com/articles/10.1186/s12711-018-0422-x. Cited 3 May 2019.

42. Gandolfi B, Alhaddad H, Abdi M, Bach LH, Creighton EK, Davis BW, et al. Applications and efficiencies of the first cat 63K DNA array. Sci Rep. 2018; 8(1):7024 Available from: http://www.nature.com/articles/s41598-018-254380 . Cited 3 May 2019.

43. Wiener P, Sánchez-Molano E, Clements DN, Woolliams JA, Haskell MJ, Blott SC. Genomic data illuminates demography, genetic structure and selection of a popular dog breed. BMC Genomics 2017;18(1):609. Available from: http://bmcgenomics.biomedcentral.com/articles/10.1186/s12864-017-3933-x. Cited 3 May 2019.

44. Druet T, Gautier M. A model-based approach to characterize individual inbreeding at both global and local genomic scales. Mol Ecol. 2017;20.

\section{Publisher's Note}

Springer Nature remains neutral with regard to jurisdictional claims in published maps and institutional affiliations.

Ready to submit your research? Choose BMC and benefit from:

- fast, convenient online submission

- thorough peer review by experienced researchers in your field

- rapid publication on acceptance

- support for research data, including large and complex data types

- gold Open Access which fosters wider collaboration and increased citations

- maximum visibility for your research: over $100 \mathrm{M}$ website views per year

At BMC, research is always in progress.

Learn more biomedcentral.com/submissions 